\title{
Adjustable Data Cleaning Towards Extracting Statistical Information
}

\author{
Argyro MAVROGIORGOU ${ }^{\mathrm{a}, 1}$, Athanasios KIOURTIS ${ }^{\mathrm{a}}$, \\ George MANIAS ${ }^{\mathrm{a}}$ and Dimosthenis KYRIAZIS ${ }^{\mathrm{a}}$ \\ a Department of Digital Systems, University of Piraeus, Greece
}

\begin{abstract}
Each device, organization, or human, is affected by the effects of Big Data. Analysing these vast amounts of data can be considered of vital importance, surrounded by many challenges. To address a portion of these challenges, a Data Cleaning approach is being proposed, designed to filter the non-important data. The functionality of the Data Cleaning is evaluated on top of Global Terrorism Data, to furtherly create policies on how terrorism is affecting national healthcare.
\end{abstract}

Keywords. Data Cleaning, Health Policies, Global Terrorism Data

\section{Introduction}

Every device or human, is both producing and surrounded by the term of data, falling under three (3) categories: Fast, Large and Complex data [1], creating numerous challenges. Since Data Cleaning improves the data quality and the analysis outcomes, this paper presents a way of effectively performing cleaning actions through four (4) steps, which are adapting their functionality based on the domain that the cleaning actions must be performed.

For Data Cleaning the authors in [2] are filling the missing values and smoothing out the noise, while in [3] they create materialized views (MVs) that pre-process and avoid complex resource intensive calculations. Furthermore, in [4] they proposed the NADEEF architecture that allows users to specify multiple types of data quality rules.

Regarding the rest of this paper, Section 2 depicts the overall proposed approach, while Section 3 includes the evaluation of the derived results, presenting our concluding remarks.

\section{Proposed Approach}

The Data Cleaning workflow comprises of four (4) steps. However, the initial basic action of the mechanism is to ingest the data from the various data sources and identify their domain, by discovering and analysing the semantics of the ingested data, and thus the Data Cleaning actions to be performed are adjusted according to the domain's nature. Afterwards, the rest of the services (Fig. 1) initiate their tasks for accomplishing all the actions related to Data Validation, Data Cleaning, Data Verification, and Data Logging.

\footnotetext{
${ }^{1}$ Corresponding Author, Argyro Mavrogiorgou, 80, M. Karaoli \& A. Dimitriou St., 18534 Piraeus, Greece; E-mail: margy@unipi.gr.
} 


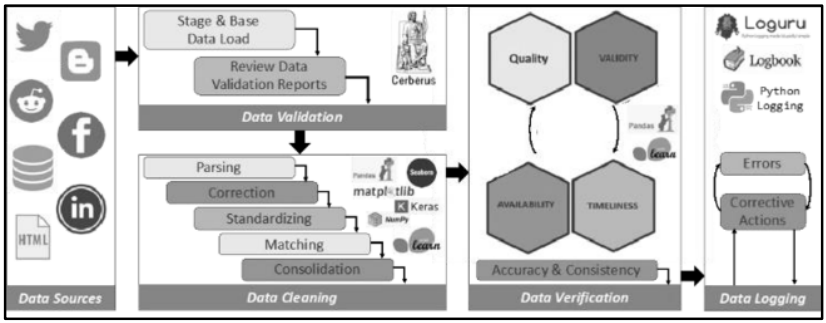

Figure 1. Data Cleaning Workflow.

\section{Evaluation and Discussion}

To evaluate the Data Cleaning approach, the described data cleaning services have been applied to the Global Terrorism Database (GTD) [5] (Fig. 2), in order to gather insights and policies regarding how global terrorism is affecting the healthcare systems of countries with increased or decreased terrorism attacks accordingly.

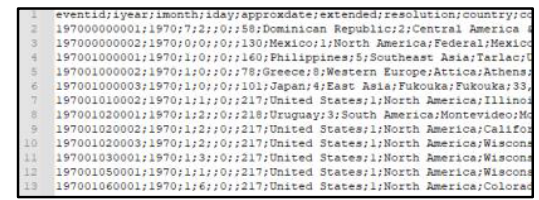

(a)

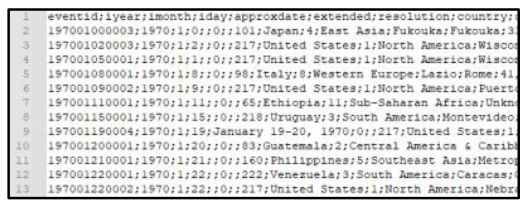

(b)

Figure 2. (a) Ingested Data, (b) Cleaned Data.

Several rows of the GTD were dropped, where, out of the 5,000 different instances, 1,563 instances were dropped after three (3) iterations of the provided mechanism. It is within our next goals to perform additional evaluation, to deploy the services in such a way to run in parallel and, respecting privacy and security concerns [6].

\section{Acknowledgment}

The research leading to the results presented in this paper has received funding from the European Union's funded Project PolicyCLOUD under grant agreement no 870675.

\section{References}

[1] Oussous A, et al.. Big Data technologies: A survey, Journal of King Saud University-Computer and Information Sciences. 2018;30(4): 431-448.

[2] Somasundaram RS, et al. Evaluation of three Simple Imputation Methods for Enhancing Preprocessing of Data with Missing Values, International Journal of Computer Applications. 2011;21(10).

[3] Saqib M, et al. Improve Data Warehouse Performance by Preprocessing and Avoidance of Complex Resource Intensive Calculations, International Journal of Computer Science Issues. 2012;9(2).

[4] Dallachiesa M, et al. NADEEF: A Commodity Data Cleaning System, ACM SIGMOD International Conference on Management of Data. 2013.

[5] Global Terrorism Database (GTD), http://ghdx.healthdata.org/record/global-terrorism-database

[6] Kiourtis A, et al. Towards a Secure Semantic Knowledge of Healthcare Data Through Structural Ontological Transformations. Joint Conf. on Knowledge-Based Software Engineering. 2018:178-188. 